\title{
Competitiveness of a Genetically Engineered Strain of Trichoderma virens
}

\author{
Mark A. Weaver · Charles M. Kenerley
}

Received: 24 October 2007 / Accepted: 31 March 2008/Published online: 18 April 2008

(C) Springer Science+Business Media B.V. 2008

\begin{abstract}
The intraspecific competitiveness of a genetically engineered strain of Trichoderma virens was assessed relative to the non-transformed, progenitor strain and an isogenic, auxotrophic strain using a replacement series design. The transformed strain was less fit, but appeared more competitive than the wild type or the auxotroph in this assay. To validate this finding and to evaluate the ability of a strain to establish in an environment occupied by a competitor another experimental approach was developed. In various treatments the transformed strain was unaffected or only slightly inhibited by a competing strain. In contrast, the wild type and the auxotrophic strain were strongly inhibited by the presence of the transformed strain. These findings support the conclusion that this genetically engineered strain is more competitive than the wild-type strain and thus may be persistent in the environment.
\end{abstract}

Keywords Biological control · Fungal competition - Intraspecific competition · Replacement series

M. A. Weaver $(\bowtie)$

Southern Weed Science Research Unit, USDA, ARS,

Stoneville, MS, USA

e-mail: mark.weaver@ars.usda.gov

C. M. Kenerley

Department of Plant Pathology and Microbiology, Texas

A\&M University, College Station, TX, USA

\section{Introduction}

Several ecological traits make Trichoderma virens an attractive candidate for two seemingly unrelated applications: bioremediation and biocontrol of plant pathogens. $T$. virens can metabolize an impressive array of substrates, including many toxins [1-4]. The species is characterized by rapid growth, abundant sporulation, and production of long-lived and durable chlamydospores [5]. Various strains produce interesting antibiotic compounds and a battery of lytic enzymes [6-12]. The mycoparasitic properties of Trichoderma spp. are well documented [e.g., 13, 14]. $T$. virens is also among the best and longest studied biocontrol fungi, with a history spanning over 70 years [15]. In this time period there have been many field releases and the fungus has been grown in large-scale fermentation with no reports of adverse non-target or human effects [16-18]. There are several ongoing efforts to genetically engineer $T$. virens to capitalize on these unique qualities and to confer new attributes [19-24].

With the possibilities of genetic engineering come two potential liabilities. One concern is efficacy. Although some of the lines of Trichoderma have been genetically altered for basic research purposes [23], most transformants are of a more applied nature. A component of successful deployment of these organisms will be their ability to compete with indigenous microflora, including wildtype Trichoderma. 
Another concern pertinent to field release of transgenic organisms is risk management. There are reports that document the performance and survival of genetically engineered microbes [24-28], mostly bacteria, and also of plants [29] and fungi [30] and their effects on other organisms [26, 31-34]. Reasonable risk management is rooted in risk assessment, which, in turn, consists of identifying and characterizing the hazard and of assessing the probability that the event of concern will occur [35-36]. Some of the hazards particular to the field deployment of transgenic microbes include unintended long-term establishment in the ecosystem, perturbation in the existing microbial community, escape of the transformant from the target area, and unwanted biological or biochemical effects. The magnitude of each of these risks is specific to the environment and organism of concern, and generalizations are not appropriate. In evaluating the probability of occurrence of any of these events, it would be helpful to have models for the fitness and competitiveness of the transformant in environments that closely mimic the environments into which they are to be deployed. However, Drobnik [35] noted that such ecological considerations are lacking and that this leaves much uncertainty in risk assessment.

If we rely on classical and simple ecological models regarding density dependent population regulation [36-38], we might anticipate that the growth of an introduced organism will be constrained by the presence of existing strains, already present at or near the carrying capacity of the environment. Countering this assumption, we have reason to believe that populations of $T$. virens are not always regulated in a classical, density dependent fashion [39].

We might also lower our estimate of the probability of these risks if we assume, like others [40, 41], that transformants are less competitive than the wildtype progenitor strains. To date we have no data to test this hypothesis in this system. Because Trichoderma is so common, and distributed so widely, competition with wild-type strains should be a component of risk assessment.

To better understand the ecology of transgenic fungi, we investigated the fitness and competitiveness of one strain of $T$. virens that had been transformed with an antibiotic marker and a gene encoding an organophosphate hydrolase. Data from this system will provide a basis for more realistic predictions of the population dynamics of transformants in competition with wild-type populations. This information will better guide efforts at risk assessment.

\section{Materials and Methods}

\section{Strains and Cultural Conditions}

Wild-type Trichoderma virens strain Gv29-8 was isolated from agricultural soil near College Station, TX [42], and maintained on Potato dextrose agar (PDA; Difco Laboratories). The genetically engineered strain, GvT6, was constructed by transformation and heterologous integration of the plasmid pCL1, which included the hygromycin resistance gene, hyg $B$, and a gene encoding organophosphate hydrolase (OPH), opd. GvT6 was selected based on high expression levels of the OPH and stable hygromycin resistance [43]. Mutant strain Tv10.4 was created by a single point mutation in the $\arg 2$ gene, resulting in an inability to grow on media lacking arginine [20]. Conidia were collected by scraping one week old PDA cultures flooded with water and filtering the suspension through Miracloth (Calbiochem). Experiments were on solidified Vogel's minimal medium [44] supplemented with $1.5 \%$ sucrose and $2 \mathrm{mM}$ arginine (VMS+arg).

\section{Production of Alginate Prills}

Conidia of T. virens were incorporated at a rate of $10^{8}$ conidia per gram of solution containing $1 \%$ sodium alginate (medium viscosity, Sigma A2033), 20\% polyethylene glycol (PEG 8000) and 2\% ground wheat bran (all on w/w basis) [43, 45]. The solution was allowed to drip through large orifice pipette tips into a $0.25 \mathrm{M}$ solution of calcium chloride. The resulting prills were spread over plastic mesh, dried overnight in a laminar flow hood, and stored at $4{ }^{\circ} \mathrm{C}$.

\section{de Wit Competition Experiment}

Conidia of strain Gv29-8, GvT6, or Tv10.4 or a mixture of two of these strains were added to $10 \mathrm{~mL}$ molten VMS + arg and aliquoted to $60 \mathrm{~mm}$ polystyrene petri dishes. The total density was held constant at $10^{6}$ conidia per dish. Treatments included monocultures of each strain and nine intermediate mixtures 
of two strains in predetermined ratios from 1:9 to 9:1. After 4 days' incubation the cultures were blended in water and serial dilutions spread on PDA, VMS, and PDA amended with $100 \mathrm{mg} / \mathrm{l}$ hygromycin (Calbiochem) (PDA + hyg). After 2 to 4 days' incubation the number of colony forming units (CFU) was determined. The source of each CFU could be a conidium or a hyphal fragment, but microscopic examination revealed that nearly all were conidia. Each experiment included three or five replications and the experiment was repeated three times. To assess the competitiveness of each strain the yield of that strain, as measured in a competitive interaction, was divided by the yield of that strain grown as a monoculture to derive a "relative yield." Data were analyzed by lack of fit regression analysis, a test to determine if deviations from the null model were statistically significant (Function: Analyze-Fit Model-Lack of Fit JMP Version 7, SAS Institute).

After 4 days, the VMS + arg plates were blended and the populations were measured. The total population sizes were determined by counting colony forming units (CFU) per plate on PDA. The population was partitioned to the two strains by plating on selective media. Only GvT6 could grow on PDA + hyg; only Gv29-8 and GvT6 could grow on VMS.

The traditional analysis of data from a replacement series involves converting the population sizes to "relative yields." That is, the observed population at any point along the replacement series is divided by the population size of that strain when grown in monoculture. The relative yield of each strain is plotted against the initial inoculum ratio. If the negative per capita effect one strain has on the fitness of the other strain is equal to the negative effects a competitor has on itself, all the points will fall along a straight line. This straight line (dashed in figures in the Results section) represents the null hypothesis that the intrastrain effects are equal to the effects from the competing strain.

\section{Invasion-Exclusion Competition Experiment}

One hundred, $10^{4}$, or $10^{6}$ conidia of strain Gv29-8, GvT6, or Tv10.4, respectively, were added to $10 \mathrm{ml}$ molten VMS + arg and dispensed into $60 \mathrm{~mm}$ polystyrene petri dishes. These conidia represent the "pulse" strain. After $5 \mathrm{~h}$, four alginate prills were placed on top of the solidified medium as the "chase" strain. After 4 days' incubation the cultures were blended in water and serial dilutions spread on PDA, VMS, and PDA + hyg. The number of CFU was determined after incubating these plates for 2-4 days. The effect of pulse strain, pulse level, chase strain, and chase level on the yield of the pulse strain and the chase strain were determined by ANOVA and multiple regression. A photograph of the plates representing a portion of the experiment is presented in Fig. 1.

\section{Results}

de Wit Competition Experiment

Figure 2 presents the results of an experiment to validate the system. Strain Tv10.4 is isogenic with Gv29-8 and differs only in a single point mutation in an arginine biosynthetic gene. The two strains grow at a similar rate and are similarly fit. The observations presented in Fig. 2 do not significantly diverge from the null hypothesis, equally competitive lines (RYT $\mathrm{F}=1.55, P=0.146 ; \mathrm{Gv} 29-8 \mathrm{~F}=1.49, P=0.164$; Tv10.4 $\mathrm{F}=0.98, P=0.461$; numerator $\mathrm{df}=9$ and denominator $\mathrm{df}=88$ in all analyses) and so we concluded that the two strains are similarly competitive. This was in contrast to the observations of GvT6 in competition with Gv29-8 (Fig. 3). In that figure, GvT6 systematically diverged above its null hypothesis line while Gv29-8 diverged below its respective lines line $(\mathrm{RYT} F=2.0, P=0.049 ; \mathrm{Gv} 29-8 \mathrm{~F}=6.88$, $P<0.0001$; GvT6 $\mathrm{F}=7.0, P<0.0001$ numerator $\mathrm{df}=9$ and denominator $\mathrm{df}=88$ in all analyses). Similar results were obtained from de Wit competition between GvT6 and Tv10.4 (Fig. 4) lines (RYT $\mathrm{F}=0.65, P=0.755 ;$ Tv10.4 $\mathrm{F}=11.7, P<0.0001$; GvT6 $\mathrm{F}=2.92, P=0.0045$ numerator $\mathrm{df}=9$ and denominator $\mathrm{df}=88$ in all analyses). The results presented here are of "relative yield" to facilitate analysis of competitive differences. The actual yield, or fitness, of GvT6 was 2.1-2.4 times less than Gv29-8 and Tv10.4 (data not shown); however, analysis of the results of the de Wit experimental system indicates it is more competitive than either non-transformed strain.

Invasion-Exclusion Experiment

If the transgenic strain, GvT6, is more competitive than the progenitor strain, Gv29-8, the results should 
Fig. 1 Invasion-exclusion experimental design. These dishes contained known quantities of conidia of strain GvT6. At the indicated time points, prills of strain Gv29-8 were added. Not shown are the reciprocal treatments containing conidia of Gv29-8 and prills of GvT6 and treatments including strain 10.4. This photo was taken after 4 days' incubation

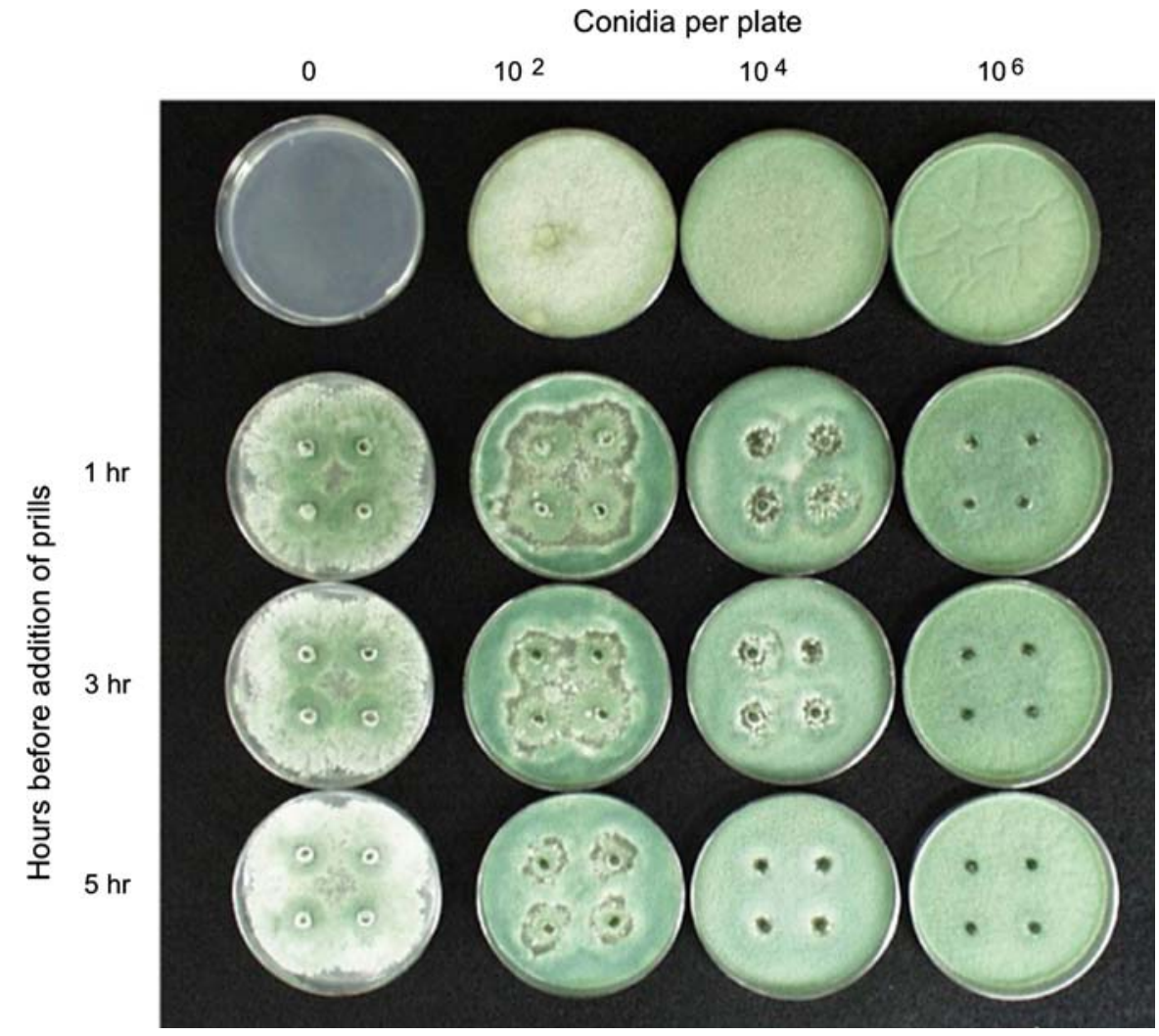

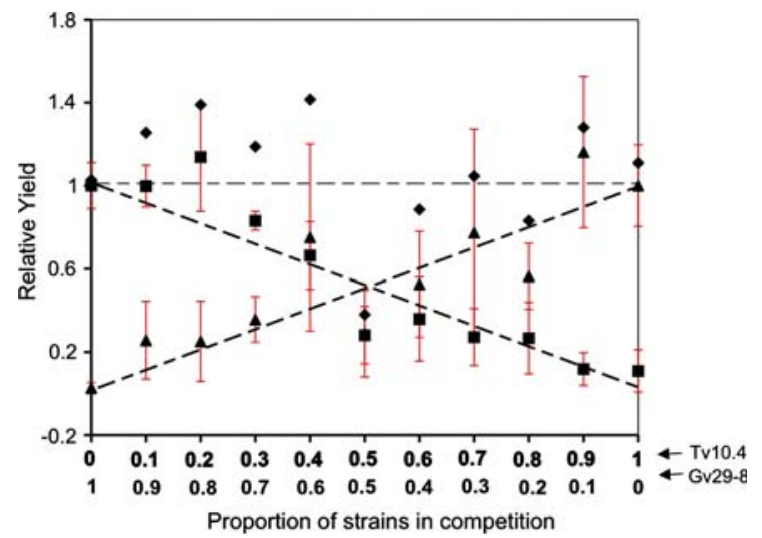

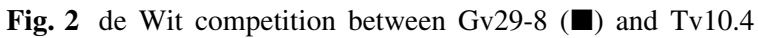
$(\boldsymbol{\Delta})$. Dashed lines represent the null hypothesis, equally competitive model. Sum of the relative yields at each ratio is given as relative yield total $(\diamond)$. Symbols show the average of three experiments, with error bars representing standard error of the mean

be discernable in other experimental systems. In the invasion-exclusion experimental approach the medium was given an inoculum "pulse" in the form of conidia in the medium and, in some treatments, a

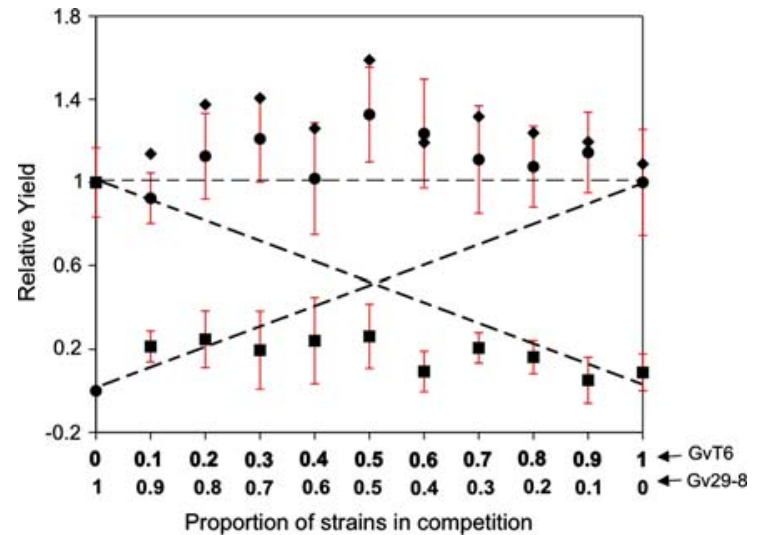

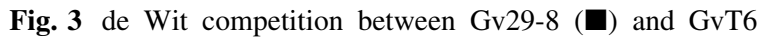
(O). Dashed lines represent the null hypothesis, equally competitive model. Sum of the relative yields at each ratio is given as relative yield total $(\diamond)$. Symbols show the average of three experiments, with error bars representing standard error of the mean

competing strain was introduced as a "chase." The time lag before addition of the chase strain did not have a statistically significant effect on the outcome of competition (effect on pulse yield $\mathrm{F}=0.0051$ 


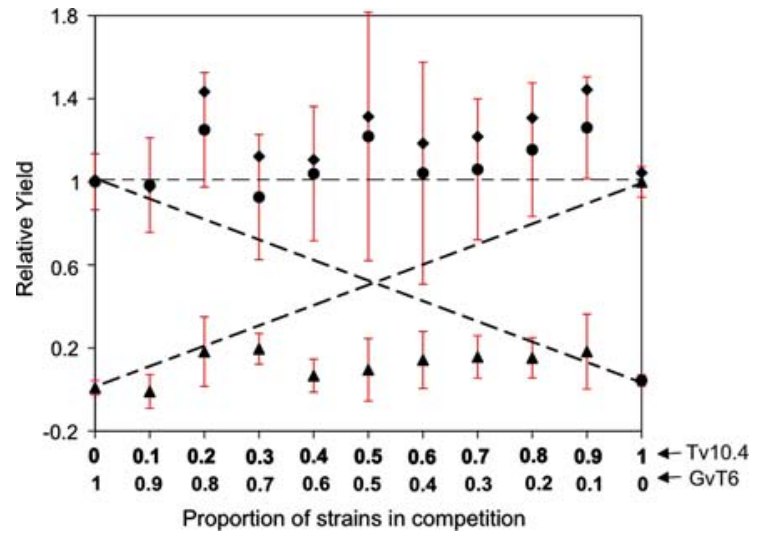

Fig. 4 de Wit competition between Tv10.4 (A) and GvT6 (O). Dashed lines represent the null hypothesis, equally competitive model. Sum of the relative yields at each ratio is given as relative yield total $(\bullet)$. Symbols show the average of three experiments, with error bars representing standard error of the mean

$\mathrm{df}=1$; effect on chase yield $\mathrm{F}=0.1130 \mathrm{df}=1$ ). The yield of each strain in competition was compared to the yield of that strain grown in monoculture (null treatment in figures). For example, Figs. 5 and 6 detail the results of competition between GvT6 prills and conidia of Gv29-8 and Tv10.4. From Fig. 5 it is apparent the GvT6 prills were inhibited, affected by the presence of a competing strain in the medium. However, this inhibition was much less than the inhibition of the competing strains by GvT6 prills (Fig. 6). In fact, in a few replicates, more colonies

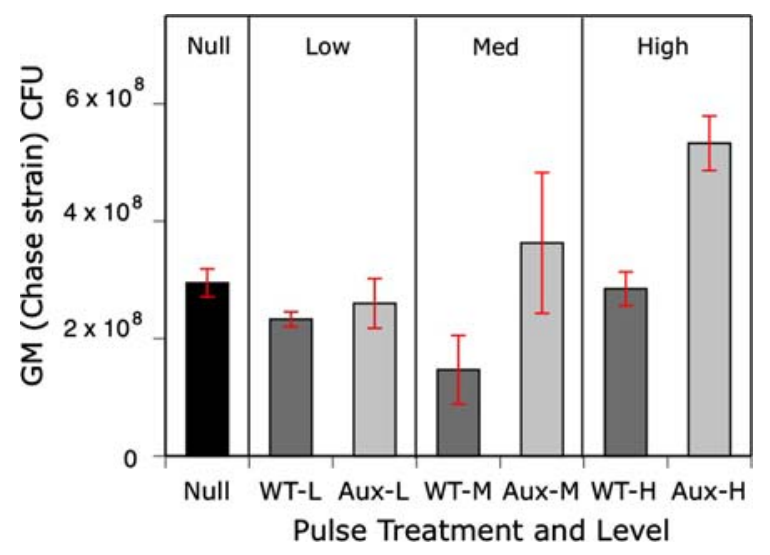

Fig. 5 Growth from prills of strain GvT6 (GM) on VMS $+\arg$ (Null) or on VMS + arg containing conidia of strain Gv29-8 (WT) or Tv10.4 (Aux). L, M, and H represent $100,10,000$, and 1,000,000 conidia per dish, respectively. Error bars indicate one standard error of the mean. "Null" indicates the growth of prills of the GM strain in monoculture

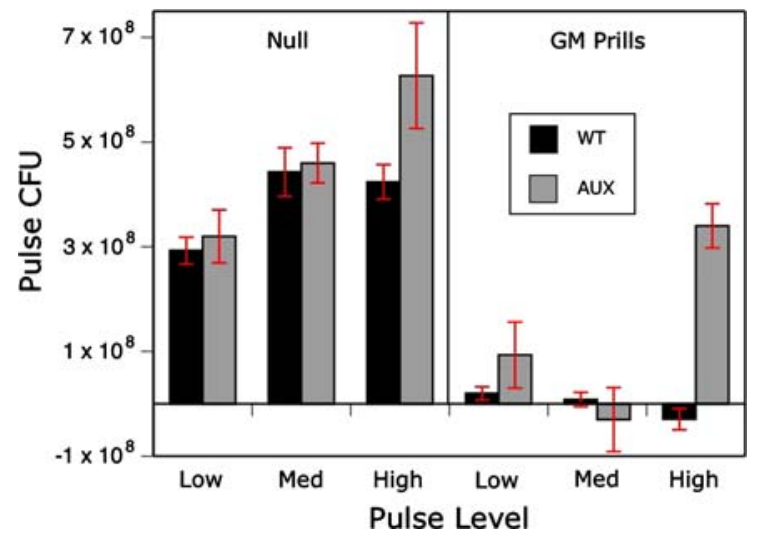

Fig. 6 Growth of conidia of strain Gv29-8 (WT) or Tv10.4 (Aux) on VMS + arg alone or under prills of strain T6 (GM). Low, Medium, and High represent 100, 10,000, and 1,000,000 conidia per dish, respectively. Error bars indicate one standard error. "Null" indicates the growth of the conidia in monoculture

were found to grow on PDA-hyg than on PDA, resulting in the appearance of a "negative" fitness in some treatments.

The reciprocal experiment, the placement of prills of Gv29-8 and Gv10.4 on conidia of GvT6, yielded different results, as indicated in Figs. 7 and 8. Figure 7 illustrates the large negative effect high concentrations of GvT6 conidia as the pulse strain had on the growth of both Gv29-8 and Tv10.4 prills, added as the chase strain. In Fig. 8 it is evident that while low concentrations of GvT6 conidia are

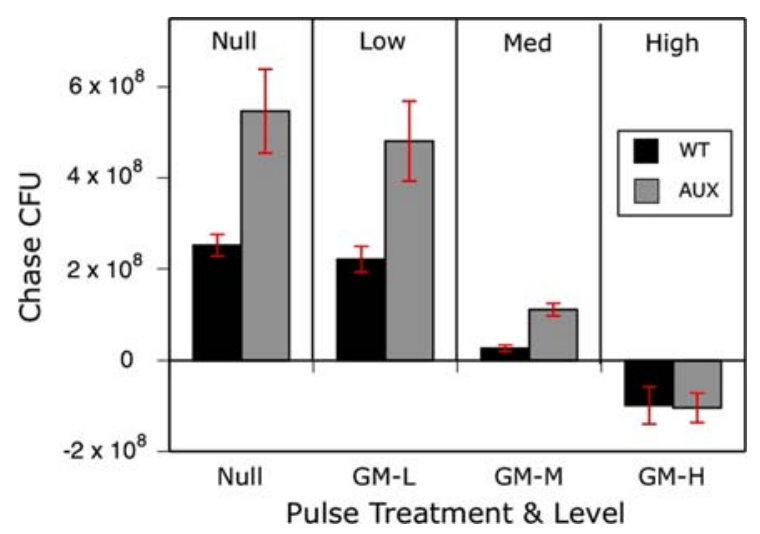

Fig. 7 Growth from prills of strain Gv29-8 (WT) or Tv10.4 (Aux) on VMS $+\arg$ (Null) or on VMS + arg containing conidia of strain GvT6 (GM). L, M, and H represent 100, 10,000, and 1,000,000 conidia per dish, respectively. Error bars indicate one standard error. "Null" indicates the growth of prills of the WT or Aux strain in monoculture 


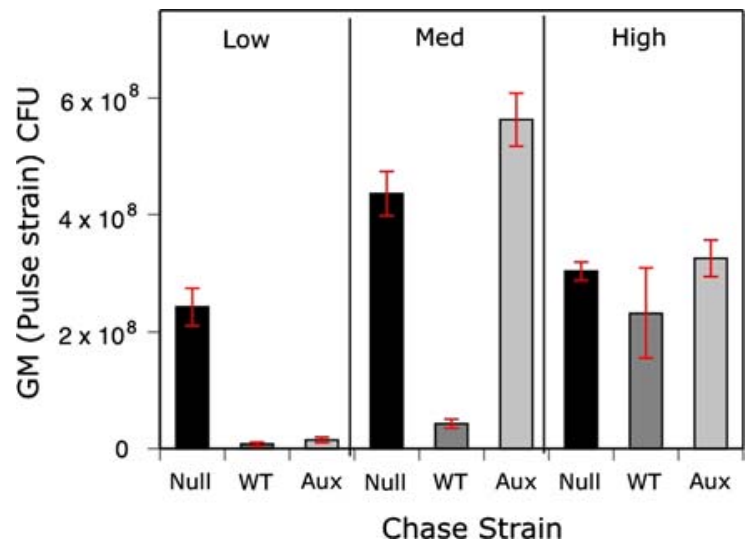

Fig. 8 Growth of conidia of strain GvT6 (GM) on VMS + arg alone or under prills of strain Gv29-8 (WT) or Tv10.4 (Aux). Low, Medium, and High represent 100, 10,000, and 1,000,000 conidia per plate, respectively, of each chase strain competitor. Error bars indicate one standard error. "Null" indicates the growth of the conidia in the absence of competition

inhibited by the competing prills, higher concentrations of conidia are unaffected by the competitors applied as chase strains.

Although the concentration of conidia present in the media clearly altered the outcome of competition, the time lags in these experiments did not have an effect $(P>\mathrm{F}=0.55)$. In contrast, pulse strain, pulse level, and chase strain were all significant in predicting the yield of the pulse or chase strain $(P>\mathrm{F}=0.05$ or less $)$.

\section{Discussion}

The results of competition experiments with the transformant, GvT6, and its wild-type progenitor, Gv29-8 (Fig 2), were unexpected. Over the years, evidence from several independent experiments, including diverse techniques, revealed that GvT6 did not grow as quickly, as Gv29-8 [21, 45, 46]. Although those tests were not designed explicitly to measure fitness (i.e., net reproductive success), and it is not essential that higher fitness be associated with greater competitiveness, it was still surprising to see the slower growing transformant exert such a strong negative per capita effect on the fitness of the wild type. Our results are consistent with the observations of the slower growth rate of the transformant, as GvT6 was less fit than Gv29-8 in both experimental systems. However, in the experiments following the de Wit approach, GvT6 was more competitive than Gv29-8 and more competitive than the mutant Tv10.4. In fact, in some treatments, the transformant was actually more fit in the presence of the wild type than when grown as a pure culture. In the pulse-chase approach GvT6 more strongly inhibited the growth of Gv29-8 and Tv10.4 than the converse.

The interaction between wild-type and the transformed $T$. virens was always a negative interaction for the wild-type strain. For the transformed strain, it was always either a neutral or a positive interaction. These positive-negative interactions are typical of predator-prey or host-pathogen interactions. This was not an anticipated outcome of intraspecific competition. In fact, we cannot find in the literature a fully appropriate term to describe the phenomenon. It is similar to the genetic concept of complementation of an auxotroph. Because the wild-type strain appears to permit a level of fitness that the transformant cannot realize in the absence of the wild-type strain, facilitation might be appropriate. Hyper-competitive might also be descriptive. It is difficult to further speculate without better understanding the mechanism that creates the phenomenon.

While the de Wit approach is a classical experimental design in ecology and has been used in numerous and diverse studies, it has been harshly criticized [48]. Many of the criticisms do not apply to our system because ours involves intraspecific competition between isogenic strains in a defined and non-selective environment where per capita fitness is known to be strongly density dependent.

Partly in response to the criticisms surrounding the de Wit design, we explored an alternative methodology. If genetically altered organisms are ever deployed in the agroecosystem, they will not be released simultaneously with other competitors in a sterile, homogenous environment. Instead, the agents will be introduced into an environment that is already colonized by other competitors, including wild-type T. virens; thus, competition will occur non-uniformly temporally and spatially. This system, it could be argued, is not simple, or pure competition. This system also involves invasiveness and the ability to ward off such invasion. Although this system is more complex and mathematically intractable, it may also be more representative of the likely outcome of field release of this transformant. 
What could account for the transformant strains' competitive ability? In view of the numerous criticisms of the de Wit replacement series, we considered the possibility that our results were an artifact of the experimental system. To minimize the likelihood of artifacts and to address the most meaningful criticisms of the de Wit replacement series, we first proved that the population was negatively regulated by density dependent mechanisms. Also to validate the experimental system we studied competition between the wild-type strain Gv29-8 and its isogenic mutant, Tv10.4. The observation that the recorded relative yield totals are linear in competition between Tv10.4 and Gv29-8, but not in competition between GvT6 and Tv10.4 or GvT6 and Gv29-8 suggests that there was a competitive imbalance in experiments that involve the transformant. The lack of any observable differences in competitiveness between Tv10.4 and Gv29-8 makes simple artifacts less plausible. The superior competitive ability of GvT6 was also independently supported in the invasion-exclusion experiments.

There may be a simple, mathematically based means of accounting for the differences in competitiveness. One deficiency of the Lotka-Volterra competition model is that it ignores complexities in life cycles. More realistic models (e.g., [49]) explicitly include stages in the model for different developmental stages. It is possible that the transformant and the wild type were actually equally competitive, in the strictest sense, but differed in their sporulation efficiency. It is also reasonable that the vegetative cells of $T$. virens are more expensive, energetically, than conidia. If this were true, then GvT6 and Gv29-8 would consume a similar quantity of resources during vegetative growth, but then, because GvT6 would produce fewer spores per unit of thallus, its overall resource consumption, per CFU (including conidia) would be higher. We had anticipated that with these two isogenic competitors, the mechanism of competition was indirect, resource-mediated competition. In this system, high resource consumption would be indistinguishable from a higher coefficient of competition. This hypothesis makes two predictions that are testable. If it is true, GvT6 should produce fewer conidia per unit of hyphae than Gv29-8. Also, in similarly designed experiments in environments that do not support sporulation the two strains should appear more equally competitive.
Whatever the mechanism, the observation that this transformant is more competitive, at least in some environments, than the parental strain has important implications. First, it challenges the assumptions regarding the competitiveness of transformants. We did not explicitly measure competition coefficients and the values would differ from one experiment to another. However it is clear that not only do they differ in magnitude for the transformant and the wild type, but they are also qualitatively different. Finally, our observations should be considered in making predictions of the population dynamics of genetically altered organisms in competition with wild-type strains. It might have seemed reasonable in a riskassessment model to anticipate that a transformant, particularly one that is less fit, might be kept in check by competition with the indigenous population. Our data instead suggest that this indigenous population might actually facilitate escape or entrenchment of the transformant.

\section{References}

1. Domsch KH, Gams W, Anderson TH. Compendium of soil fungi. New York: Academic Press; 1980.

2. Gudin C, Chater KWA. Isolation of Trichoderma harzianum (Rifai) growing on ferric hydroxide mud impregnated with gas oil. Environ Pollut. 1977;14:1-4.

3. Nevalainen H, Neethling D. The safety of Trichoderma and Gliocladium In: Harman GE, Kubicek CP, editors. Trichoderma and Gliocladium. Bristol, PA: Taylor \& Francis; 1998.

4. Papavizas GC. Trichoderma and Gliocladium: biology, ecology and potential for biocontrol. Annu Rev Phytopathol. 1985;23:23-54.

5. Klein D, Eveligh DE. Ecology of Trichoderma. In: Harman GE, Kubicek CP, editors. Trichoderma and Gliocladium. Bristol, PA: Taylor \& Francis; 1998. p. 57-74.

6. Haran S, Schickler H, Oppenheim A, Chet I. Differential expression of Trichoderma harzianum chitinases during mycoparasitism. Phytopathology. 1996;86:980-5.

7. Harman GE, Hayes CK, Lorito M, Broadway RM, Di Pietro A, Peterbauer C, Tronsmo A. Chitinolytic enzymes of Trichoderma harzianum purification of chitobiosidase and endochitinase. Phytopathology. 1993;83: 313-8.

8. Howell CR. The role of antibiosis in biocontrol. In: Harman GE, Kubicek CP, editors. Trichoderma and Gliocladium. Bristol, PA: Taylor \& Francis; 1998. p. 173-84.

9. Kim DJ, Baek JM, Uribe P, Kenerley CM, Cook DR. Cloning and characterization of multiple glycosyl hydrolase genes from Trichoderma virens. Curr Genet. 2002;40: 374-84.

10. Okuda T, Kohno J, Kishi N, Asai Y, Nishio M, Komatsubara S. Production of TMC-151, TMC-154 and TMC-171, a new 
class of antibiotics, is specific to Gliocladium roseum group. Mycoscience. 2000;41:239-53.

11. Wiest A, Grzegorski D, Xu BW, Goulard C, Rebuffat S, Ebbole DJ, Bodo B, Kenerley CM. Identification of peptaibols from Trichoderma virens and cloning of a peptaibol synthetase. J Biol Chem. 2002;277:20862-8.

12. Mischke S. A quantitative bioassay for extracellular metabolites that antagonize growth of filamentous fungi, and its use with biocontrol fungi. Mycopathologia. 1997;137:45-52.

13. Menendez AB, Godeas A. Biological control of Sclerotinia sclerotiorum attacking soybean plants. Degradation of the cell walls of this pathogen by Trichoderma harzianum (BAFC 742): Biological control of Sclerotinia sclerotiorum by Trichoderma harzianum. Mycopathologia. 1998;142: 153-60.

14. Sánchez V, Rebolledo O, Picaso RM, Cárdenas E, Córdova J, González O, Samuels GJ. In vitro antagonism of Thielaviopsis paradoxa by Trichoderma longibrachiatum. Mycopathologia. 2007;163:49-58.

15. Weindling R. Trichoderma lignorum as a parasite of other soil fungi. Phytopathology. 1932;22:837-45.

16. Harman GE. Myths and dogmas of biocontrol: changes in perceptions derived from research on Trichoderma harzianum T-22. Plant Dis. 2000;84:377-93.

17. Hjeljord L, Tronsmo A. Trichoderma and Gliocladium in biological control: An overview. In: Harman GE, Kubicek CP, editors. Trichoderma and Gliocladium. Bristol, PA: Taylor \& Francis; 1998. p. 131-51.

18. Papavizas GC, Dunn MT, Lewis JA, Beagle-Ristaino JE. Liquid fermentation technology for experimental production of biocontrol fungi. Phytopathology. 1984;74:1171-5.

19. Baek JM, Howell CR, Kenerley CM. The role of an extracellular chitinase from Trichoderma virens Gv29-8 in the biocontrol of Rhizoctonia solani. Curr Genet. 1999;35: 41-50.

20. Baek JM, Kenerley CM. The arg2 gene of Trichoderma virens: cloning and development of a homologous transformation system. Fungal Genet Biol. 1998;23:34-44.

21. Cross D. Digital imaging, modeling of the growth of Trichoderma virens strains designed to degrade methyl parathion [Dissertation]. College Station, TX: Texas A\&M University; 2000.

22. Lorito M, Scala F, Zoina A, Woo S. Enhancing biocontrol of fungal pests by exploiting the Trichoderma genome. In: Varro M, Gressel J, Butt T, Harman GE, Pilgerman A, Leger RS, Nuss D, editors. Enhancing Biocontrol Agents and Handling Risks. Amsterdam: IOS Press; 2001. p. 248-59.

23. Bae YS, Knudsen GR. Cotransformation of Trichoderma harzianum with beta-glucuronidase and green fluorescent protein genes provides a useful tool for monitoring fungal growth and activity in natural soils. Appl Environ Microbiol. 2000;66:810-5.

24. Djonovic S, Vittone G, Mendoza-Herrera A, Kenerley CM. Enhanced biocontrol activity of Trichoderma virens transformants constitutively co-expressing $\beta$-1,3- and $\beta$-1,6glucanase genes. Molecular Plant Pathol. 2007;8:469-80.

25. de Leij FAAM, Sutton EJ, Whipps JM, Fenlon JS, Lynch JM. Impact of field release of genetically modified Pseudomonas fluorescens on indigenous microbial populations of wheat. Appl Environ Microbiol. 1995;61:3443-53.
26. Mahaffee WF, Kloepper JW. Bacterial communities of the rhizosphere and endorhiza associated with field-grown cucumber plants inoculated with a plant growth-promoting rhizobacterium or its genetically modified derivative. Can J Microbiol. 1997;43:344-53.

27. Naseby DC, Lynch JM. Establishment and impact of Pseudomonas fluorescens genetically modified for lactose utilization and kanamycin resistance in the rhizosphere of pea. J Appl Microbiol. 1998a;84:169-75.

28. Naseby DC, Lynch JM. Impact of wild-type and genetically modified Pseudomonas fluorescens on soil enzyme activities and microbial population structure in the rhizosphere of pea. Mol Ecol. 1998b;7:617-25.

29. Giddings GD, Hamilton NR, Sackville HMD. The release of genetically modified grasses. Part 1: Pollen dispersal to traps in Lolium perenne. Theor Appl Genet. 1997;94: 1000-6.

30. Bowen JK, Crowhurst RN, Templeton MD, Stewart A. Molecular markers for a Trichoderma harzianum biological control agent: Introduction of the hygromycin B resistance gene and the beta-glucuronidase gene by transformation. N Z J Crop Hortic Sci. 1996;24:219-28.

31. Girlanda M, Perotto S, Moenne-Loccoz Y, Bergero R, Lazzari A, Defago G, Bonfante P, Luppi AM. Impact of biocontrol Pseudomonas fluorescens CHA0 and a genetically modified derivative on the diversity of culturable fungi in the cucumber rhizosphere. Appl Environ Microbiol. 2001;67:1851-64.

32. Jesse LCH, Obrycki JJ. Field deposition of Bt transgenic corn pollen: Lethal effects on the monarch butterfly. Oecologia. 2000;125:241-8.

33. Schuler TH, Denholm I, Jouanin L, Clark SJ, Clark AJ, Poppy GM. Population-scale laboratory studies of the effect of transgenic plants on nontarget insects. Molec Ecol. 2001;10:1845-53.

34. Tschenn J, Losey JE, Jesse LH, Obrycki JJ, Hufbauer R. Effects of corn plants and corn pollen on monarch butterfly (Lepidoptera:Danaidae) oviposition behavior. Environ Entomol. 2001;30:495-500.

35. Drobnik J. Genetically modified organisms (GMO) in bioremediation and legislation. Int Biodeterior Biodegradation. 1999;44:3-6.

36. Gustafsson K, Jansson JK. Ecological risk assessment of the deliberate release of genetically modified microorganisms. Ambio. 1993;22:236-42.

37. Nicholson AJ. Competition for food amongst Lucilia cuprina larvae. Proceedings of the Eighth International Congress of Entomology, Stockholm. 1948. p. 277-81.

38. Ricker WE. Computation and interpretation of biological statistics of fish populations. Bull Fisheries Res Board Can. 1975;191:1-382.

39. Weaver MA, Kenerley CM. Density independent population dynamics by Trichoderma virens in soil and defined substrates. Biocontrol Sci Technol. 2005;15:847-57.

40. Brill WJ. Safety concerns and genetic engineering in agriculture. Science. 1985;227:381-4.

41. Davis BD. Bacterial domestication: underlying assumptions. Science. 1987;235:1329-35.

42. Park YH, Kenerley CM, Stack JP. Inoculum dynamics of Gliocladium virens associated with roots of cotton seedlings. Microb Ecol. 1992;23:169-79. 
43. Dave KI, Lauriano CM, Xu B, Wild JR, Kenerley CM. Expression of organophosphate hydrolase in the filamentous fungus Gliocladium virens. Appl Microbiol Biotechnol. 1994;41:352-8.

44. Vogel HJ. A convenient growth medium for Neurospora (Medium N). Microbiol Genet Bull. 1956;13:42-3.

45. Daigle DJ, Cotty PJ. The effect of sterilization, $\mathrm{pH}$, filler and spore inoculum concentration on the preparation of alginate pellets. Biocontrol Sci Technol. 1997;7:3-10.

46. Classen JJ, Whittaker AD, Kenerley CM. Fractal analysis of subsurface growth of a genetically modified strain of
Gliocladium virens and its parental strain. Trans Am Soc Agric Eng. 1996;29:2271-6.

47. Weaver MA, Vedenyapina E, Kenerley CM. Fitness, persistence, and responsiveness of a genetically engineered strain of Trichoderma virens in soil mesocosms. Appl Soil Ecol. 2005;29:125-34.

48. Connolly J. On difficulties with replacement-series methodology in mixture experiments. J App Ecol. 1986;23:125-37.

49. Newton MR, Kinkel LL, Leonard KJ. Competition and density-dependent fitness in a plant parasitic fungus. Ecology. 1997;78:1774-84. 